\title{
A REAL PART THEOREM FOR THE HIGHER DERIVATIVES OF ANALYTIC FUNCTIONS IN THE UNIT DISK
}

\author{
DAVID KALAJ AND NOAM D. ELKIES
}

\begin{abstract}
Let $n$ be a positive integer. Let $\mathbf{U}$ be the unit disk, $p \geq 1$ and let $h^{p}(\mathbf{U})$ be the Hardy space of harmonic functions. Kresin and Maz'ya in a recent paper found a representation for the function $H_{n, p}(z)$ in the inequality

$$
\left|f^{(n)}(z)\right| \leq H_{n, p}(z)\left\|\Re\left(f-\mathcal{P}_{l}\right)\right\|_{h^{p}(\mathbf{U})}, \quad \Re f \in h^{p}(\mathbf{U}), z \in \mathbf{U},
$$

where $\mathcal{P}_{l}$ is a polynomial of degree $l \leq n-1$. We find or represent the sharp constant $C_{p, n}$ in the inequality $H_{n, p}(z) \leq \frac{C_{p, n}}{\left(1-|z|^{2}\right)^{1 / p+n}}$. This extends a recent result of Kalaj and Marković, where only the case $n=1$ was considered. As a corollary, an inequality for the modulus of $n-t h$ derivative of an analytic function defined in a complex domain with the bounded real part is obtained. This result improves a recent result of Kresin and Maz'ya.
\end{abstract}

\section{Introduction AND StATEMENT OF the RESUlts}

A harmonic function $f$ defined in the unit disk $\mathbf{U}$ of the complex plane $\mathbf{C}$ belongs to the harmonic Hardy class $h^{p}=h^{p}(\mathbf{U}), 1 \leq p<\infty$ if the following growth condition is satisfied

$$
\|f\|_{h^{p}}:=\left(\sup _{0<r<1} \int_{\mathbf{T}}\left|f\left(r e^{i t}\right)\right|^{p} d t\right)^{1 / p}<\infty
$$

where $\mathbf{T}$ is the unit circle in the complex plane $\mathbf{C}$. The space $h^{\infty}(\mathbf{U})$ consists of all bounded harmonic functions.

If $f \in h^{p}(\mathbf{U})$, then there exists the finite radial limit

$$
\lim _{r \rightarrow 1^{-}} f(r \zeta)=f^{*}(\zeta)(\text { a.e. on } \mathbf{T})
$$

and the boundary function $f^{*}$ belong to the space $L^{p}(\mathbf{T})$ of $p$-integrable functions on the circle.

It is well known that a harmonic function $f$ in the Hardy class $h^{p}(\mathbf{U})$ can be represented as the Poisson integral

$$
f(z)=\int_{\mathbf{T}} P(z, \zeta) d \mu(\zeta), z \in \mathbf{U}
$$

where

$$
P(z, \zeta)=\frac{1-|z|^{2}}{|z-\zeta|^{2}}, z \in \mathbf{U}, \zeta \in \mathbf{T}
$$

is the Poisson kernel and $\mu$ is a complex Borel measure. In the case $p>1$ this measure is absolutely continuous with respect to the Lebesgue measure and

1991 Mathematics Subject Classification. Primary 31A05; Secondary 42B30 .

Key words and phrases. Harmonic functions, Bloch functions, Hardy spaces. 
$d \mu(\zeta)=f^{*}(\zeta) d \sigma(\zeta)$. Here $d \sigma$ is Lebesgue probability measure in the unit circle. Moreover we have

$$
\|f\|_{h^{p}}=\left\|f^{*}\right\|_{p}, \quad p>1
$$

and

$$
\|f\|_{h^{1}}=\|\mu\|
$$

where we denote by $\|\mu\|$ the total variation of the measure $\mu$.

For previous facts we refer to the book [1, Chapter 6]. In the sequel for $p \geq 1$ and $m$ a positive integer, as in [10] we use the notation

$$
E_{m, p}(\Re f):=\inf _{\mathcal{P} \in \mathfrak{P}_{m}}\|\Re(f-\mathcal{P})\|_{h^{p}}
$$

for the best approximation of $\Re f$ by the real part of algebraic polynomials in the $h^{p}(\mathbf{U})$-norm, where $\mathfrak{P}_{m}$ is the set of all algebraic polynomials of degree at most $m$.

The starting position of this paper is the following proposition of Maz'ya and Kresin [10, Proposition 5.1].

Proposition 1.1. Let $f$ be analytic on $\mathbf{U}$ with $\Re f \in h^{p}(\mathbf{U}), 1 \leq p \leq \infty$. Further, let $n \geq 1$, and let $\mathcal{P}_{l}$ be a polynomial of degree $l \leq n-1$. Then for any fixed point $z,|z|=r<1$, the inequality

$$
\left|f^{(n)}(z)\right| \leq H_{n, p}(r)\left\|\Re\left(f-\mathcal{P}_{l}\right)\right\|_{h^{p}}
$$

holds with the sharp factor

$$
H_{n, p}(r)=\frac{n !}{\pi} \sup _{\alpha}\left\{\int_{|\zeta|=1}\left|\Re \frac{\zeta e^{i \alpha}}{(\zeta-r)^{n+1}}\right|^{q}|d \zeta|\right\}^{1 / q}
$$

and $1 / q+1 / p=1$. In particular

$$
\left|f^{(n)}(z)\right| \leq H_{n, p}(r) E_{n-1, p}(\Re f) .
$$

For $p=2(q=2)$ and $p=1(q=\infty)$ the function $H_{n, p}(r)$ has been calculated explicitly in [10. We refer to [10] for the connection of (1.3) and the famous Hadamard-Borel-Carathéodory inequality:

$$
|f(z)-f(0)| \leq \frac{2}{1-|z|^{2}} \sup _{|\zeta|<1} \Re[f(\zeta)-f(0)] .
$$

The aim of this paper is to obtain some explicit estimations of $H_{n, p}(r)$ for general $p$. The results of this paper are

Theorem 1.2 (Main theorem). Let $1 \leq p \leq \infty$ and let $q$ be its conjugate. Let $f$ be analytic on the unit disk $\mathbf{U}$ with $\Re f \in h^{p}(\mathbf{U}), 1 \leq p \leq \infty$. Further, let $n \geq 1$, and let $\mathcal{P}_{l}$ be a polynomial of degree $l \leq n-1$. We have the following sharp inequality

$$
\left|f^{(n)}(z)\right| \leq C_{p, n}\left(1-r^{2}\right)^{-1 / p-n}\left\|\Re\left(f-\mathcal{P}_{l}\right)\right\|_{h^{p}},
$$

where

$$
C_{p, n}=\frac{n !}{\pi} 2^{n+1-1 / q} \max _{0 \leq \beta \leq \pi / 2} F_{q}^{1 / q}(\beta)
$$

and

$$
F_{q}(\beta)=\int_{0}^{\pi}\left|\sin ^{(n+1)-2 / q} v \cos \left[v(n+1)+\beta-\frac{\pi}{2}(n-1)\right]\right|^{q} d v .
$$

In particular

$$
\left|f^{(n)}(z)\right| \leq C_{p, n}\left(1-r^{2}\right)^{-1 / p-n} E_{n-1, p}(\Re f) .
$$


Remark 1.3. In connection with Theorem 1.2, we conjecture that (c.f. Conjecture 4.1)

$$
\max _{0 \leq \beta \leq \pi / 2} F_{q}(\beta)=\max \left\{F_{q}(0), F_{q}(\pi / 2)\right\} .
$$

We have the solution for $q=1$ presented in Theorem 1.4. We list some known partial solutions.

- The Hilbert case (see [10, Eq. 5.5.3] or [11, Eq. $\left.(6.1 .4)_{2}\right]$ ): for $q=2$ and all $n$, the corresponding function is

$$
F_{q}(\beta)=\frac{2^{n}}{\pi^{3 / 4}} \sqrt{\frac{\Gamma[1 / 2+n]}{\Gamma[1+n]}} .
$$

- For $q=\infty$ and all $n$ ([10, Eq. 5.4.2] for $\gamma=1$ ), $F_{q}(\beta)=2^{n+1}$.

- For $n=1$ and all $q$, (see [8]) we have

$$
\max _{0 \leq \beta \leq \pi / 2} F_{q}(\beta)=\left\{\begin{array}{ll}
F_{q}(0), & \text { if } q>2 \\
F_{q}(\pi / 2), & \text { if } q \leq 2
\end{array} .\right.
$$

We also refer to related sharp inequalities for the derivatives of analytic functions defined in the unit disk 13 .

Theorem 1.4. Let $f$ be analytic on the unit disk $\mathbf{U}$ with bounded real part $\Re f$ and assume that $\beta \in[0, \pi]$. Then

$$
\left|f^{(n)}(z)\right| \leq C_{n}\left(1-|z|^{2}\right)^{-n} \mathcal{O}_{n, \Re f}(\mathbf{U}),
$$

where

$$
\begin{gathered}
C_{n}= \begin{cases}\frac{1}{n \pi} \frac{((2 m) !)^{2}}{(m !)^{2}}, & \text { if } n=2 m-1 ; \\
\frac{n !}{\pi} \max \{F(\beta): 0 \leq \beta \leq \pi\} & \text { if } n=2 m\end{cases} \\
F(\beta)=\frac{2}{n} \sum_{k=1}^{n+1} \sin ^{n+1} \frac{k \pi-\beta}{n+1}
\end{gathered}
$$

and

$$
\mathcal{O}_{n, \Re f}(\mathbf{U})=\inf _{\mathcal{P} \in \mathfrak{P}_{n-1}} \mathcal{O}_{\Re(f-P)}(\mathbf{U})
$$

and $\mathcal{O}_{\Re f}(\mathbf{U})$ is the oscillation of $\Re f$ on the unit disk $\mathbf{U}$.

Remark 1.5. If $w=\Re f$ is a real harmonic function, where $f$ is an analytic function defined on the unit disk, then the Bloch constant of $w$ is defined by

$$
\beta_{w}=\sup _{z \in \mathbf{U}}\left(1-|z|^{2}\right)|\nabla w(z)|=\sup _{z \in \mathbf{U}}\left(1-|z|^{2}\right)\left|f^{\prime}(z)\right|
$$

and is less than or equal to $C_{1}=4 / \pi$ provided that the oscillation of $w$ in the unit disk is $\leq 1$. This particular case is well known in the literature see e.g. 3, 6, 7]. In a similar manner we define the Bloch constant of order $n$ of a harmonic function $w=\Re f$ :

$$
\beta_{n, w}=\sup _{z \in \mathbf{U}}\left(1-|z|^{2}\right)^{n}\left|f^{(n)}(z)\right|
$$

and by the previous corollary we find out that $\beta_{n, w} \leq C_{n}$ provided $n$ is an odd integer and the oscillation $\mathcal{O}_{n, \Re f}(\mathbf{U})$ is at most 1 .

The following theorem improves one of the main results in [9] (see [9, Corollary 7.1$])$. 
Corollary 1.6. Let $\Omega$ be a subdomain of C. Let $z \in \Omega$, and assume that $a_{z} \in$ $\partial \Omega$ such that $\left|z-a_{z}\right|=d_{z}=\operatorname{dist}(z, \partial \Omega)$ and that $\left[\zeta, a_{z}\right]$ is the maximal interval containing $z$ with $d_{\zeta}=\left|\zeta-a_{z}\right|$. Let $f$ be a holomorphic function in $\Omega$ with its real part in Lebesgue space $L^{\infty}(\Omega)$ and let $\|\operatorname{Re} f\|_{L^{\infty}(\Omega)} \leq 1$. Then the inequality

$$
d_{z}^{n}\left|f^{(n)}(z)\right| \leq \frac{C_{n}}{\left(2-d_{z} / d_{\zeta}\right)^{n}}, z \in \Omega
$$

holds with $C_{n}:=C_{\infty, n}$ defined in (1.6). In particular,

$$
d_{z}^{2 m-1}\left|f^{(2 m-1)}(z)\right| \leq \frac{1}{n \pi} \frac{((2 m) !)^{2}}{(m !)^{2}} \frac{1}{\left(2-d_{z} / d_{\zeta}\right)^{n}}, \quad z \in \Omega .
$$

Proof. Let $z \in \Omega$ and assume that $\zeta \in \Omega$ satisfies the condition of the theorem. Then $D_{\zeta}:=\left\{w:|w-\zeta|<d_{\zeta}\right\} \subset \Omega$. Define $g(w)=f\left(\zeta+w d_{\zeta}\right), w \in \mathbf{U}$. Then $\operatorname{Re} g \in h^{\infty}(\mathbf{U})$ and $g^{(n)}(w)=d_{\zeta}^{n} f^{(n)}\left(\zeta+w d_{\zeta}\right)$. By the maximum principle we have

$$
\|\operatorname{Re} f\|_{h^{\infty}\left(D_{\zeta}\right)} \leq\|\operatorname{Re} f\|_{L^{\infty}(\Omega)} .
$$

By applying Theorem 1.2 and Theorem 1.4 to $g$ we have

$$
\left(1-|w|^{2}\right)^{n} d_{\zeta}^{n}\left|f^{(n)}\left(\zeta+w d_{\zeta}\right)\right| \leq C_{n} .
$$

As $z \in\left[\zeta, a_{z}\right]$ it follows that $z=\zeta+s\left(a_{z}-\zeta\right)=\zeta+w d_{\zeta}$, where $w=s e^{i \phi} \in \mathbf{U}$. Since $d_{\zeta}=(1-s)^{-1} d_{z}$, and $|w|=\left|(z-\zeta) / d_{\zeta}\right|=s=\left(d_{\zeta}-d_{z}\right) / d_{z}$ we obtain that

$$
\left(1-s^{2}\right)^{n}(1-s)^{-n} d_{z}^{n}\left|f^{(n)}(z)\right| \leq C_{n}
$$

and

$$
d_{z}^{n}\left|f^{(n)}(z)\right| \leq C_{n} \frac{1}{(1+s)^{n}}=C_{n} \frac{1}{\left(2-d_{z} / d_{\zeta}\right)^{n}} .
$$

Remark 1.7. In [9, Corollary 7.1] Kresin and Maz'ya proved that

$$
\lim _{\epsilon \rightarrow 0^{+}} \sup _{z: d_{z}=\epsilon} d_{z}^{2 m-1}\left|f^{(2 m-1)}(z)\right| \leq 2^{-n} C_{n},
$$

under the condition that $\Omega$ is a planar domain with certain smoothness condition on the boundary, namely assuming that there is $r>0$ such that for $a \in \partial \Omega$, there is a disk $D_{a} \subset \Omega$ of radius $r$ with $a \in \overline{D_{a}}$. Then $d_{\zeta} \geq r$ for $z \in \Omega$. The inequality (1.11) follows by using (1.9) and letting $\epsilon \rightarrow 0$.

\section{Proof of Theorem 1.2}

In view of (1.4), we deal with the function

$$
I_{\alpha}(r)=\int_{0}^{2 \pi}\left|\Re \frac{e^{i(\alpha+t)}}{\left(r-e^{i t}\right)^{n+1}}\right|^{q} d t, \quad 0 \leq r<1 .
$$

By making use of the change

we obtain

$$
e^{i t}=\frac{r-e^{i s}}{1-r e^{i s}},
$$

$$
d t=\frac{1-r^{2}}{\left|1-r e^{i s}\right|^{2}} d s
$$

and

$$
r-e^{i t}=\frac{\left(1-r^{2}\right) e^{i s}}{1-r e^{i s}} .
$$


We arrive at the integral

$$
\begin{aligned}
I_{\alpha}(r) & =\int_{0}^{2 \pi}\left(1-r^{2}\right)^{1-q-n q}\left(1+r^{2}-2 r \cos s\right)^{-1+q}\left|\Re\left[e^{i(\alpha+s)}\left(e^{i s}-r\right)^{-1+n}\right]\right|^{q} d s \\
& =\left(1-r^{2}\right)^{1-q-n q} \int_{0}^{2 \pi} f_{\alpha}\left(r, e^{i s}\right) d s
\end{aligned}
$$

where

$$
f_{\alpha}\left(z, e^{i s}\right)=\left|\Re\left[e^{i(\alpha+s)}\left(z-e^{i s}\right)^{n-1}\right]\right|^{q}\left|z-e^{i s}\right|^{2 q-2} .
$$

In order to continue, let's prove first two lemmas.

Lemma 2.1. $f_{\alpha}$ is subharmonic in $z$.

Proof. We refer to [12, Chapter 4] and [5, Chapter I $\S 6]$ for some basic properties of subharmonic functions. Recall that a continuous function $g$ defined on a region $G \subset \mathbf{C}$ is subharmonic if for all $w_{0} \in G$ there exists $\varepsilon>0$ such that

$$
g\left(w_{0}\right) \leq \frac{1}{2 \pi} \int_{0}^{2 \pi} g\left(w_{0}+r e^{i t}\right) d t, \quad 0<r<\varepsilon .
$$

If $g\left(w_{0}\right)=0$, since $g$ is non-negative, then (2.2) holds. If $g\left(w_{0}\right)>0$, then there exists a neighborhood $U$ of $w_{0}$ such that $g$ is of class $C^{2}(U)$ and $g(w)>0 w \in U$. Thus if $g$ is $C^{2}$ where it is positive, then it is enough to check that the Laplacian is non-negative there.

Let $w=e^{i \frac{\alpha+s}{n-1}} z-e^{i s-i \frac{(\alpha+s)}{n-1}}$ and define

$$
g(w):=f_{\alpha}\left(z, e^{i s}\right)=\left|\Re\left[w^{n-1}\right]\right|^{q}|w|^{2 q-2} .
$$

Assume that $\Re\left(w^{n-1}\right)>0$. Then

$$
g_{w}=2^{-q} \bar{w}(\bar{w} w)^{q-4}\left(\bar{w}^{n-1}+w^{n-1}\right)^{q-1}\left[(-1+q) \bar{w}^{n} w+(-1+n q) \bar{w} w^{n}\right] .
$$

Further

$$
\begin{aligned}
g_{w \bar{w}} & =\frac{(q-1)(\bar{w} w)^{q-4}\left(\bar{w}^{n-1}+w^{n-1}\right)^{q-2}}{2^{q+2}} \\
& \times\left((-1+n q) \bar{w}^{2 n} w^{2}+(-1+n q) \bar{w}^{2} w^{2 n}+\left(-2+q+n^{2} q\right) \bar{w}^{1+n} w^{1+n}\right) .
\end{aligned}
$$

Observe next that

$$
\frac{(q-1)(\bar{w} w)^{q-4}\left(\bar{w}^{n-1}+w^{n-1}\right)^{q-2}}{2^{q+2}}=\frac{(q-1)|w|^{2 q-8}\left(\Re w^{n-1}\right)^{q-2}}{16} \geq 0
$$

and

$$
\begin{aligned}
(-1 & +n q) \bar{w}^{2 n} w^{2}+(-1+n q) \bar{w}^{2} w^{2 n}+\left(-2+q+n^{2} q\right) \bar{w}^{1+n} w^{1+n} \\
& =\left(-2+q+n^{2} q\right)|w|^{2 n+2}-2(-1+n q) \Re\left[\bar{w}^{2} w^{2 n}\right] \\
& \geq q(n-1)^{2}|w|^{2 n+2} \geq 0 .
\end{aligned}
$$

Similarly we treat the case $\Re\left(w^{n-1}\right)<0$. Therefore $\Delta g=4 g_{w \bar{w}} \geq 0$ for $\Re\left(w^{n-1}\right) \neq$ 0 . This implies that $g$ is subharmonic in the whole of $\mathbf{C}$. Since

$$
\left.f_{\alpha}\left(z, e^{i s}\right)\right)=g\left(e^{i \frac{\alpha+s}{n-1}} z-e^{i s-i \frac{(\alpha+s)}{n-1}}\right)
$$

we have that $\Delta f_{\alpha}\left(z, e^{i s}\right)=\Delta g(a z+b)$ which implies that $z \rightarrow f_{\alpha}\left(z, e^{i s}\right)$ is subharmonic. 
Lemma 2.2. For $\alpha \in[0, \pi]$ we have

$$
\begin{aligned}
I_{\alpha}(r) & \leq\left(1-r^{2}\right)^{1-q-n q} \max _{0 \leq t \leq 2 \pi} \int_{0}^{2 \pi} f_{\alpha}\left(e^{i t}, e^{i s}\right) d s \\
& =\left(1-r^{2}\right)^{1-q-n q} \int_{0}^{2 \pi} f_{\beta}\left(1, e^{i s}\right) d s,
\end{aligned}
$$

for some $\beta$ possibly different from $\alpha$.

Proof. Since $z \rightarrow f_{\alpha}\left(z, e^{i t}\right)$ is subharmonic in $|z|<1$, and $(t, z) \rightarrow f_{\alpha}\left(z, e^{i t}\right)$ is continuous in $[0, \pi] \times \mathbf{U}$, then the integral mean $I(z)=\int_{0}^{2 \pi} f_{\alpha}\left(z, e^{i s}\right) d s$ is a subharmonic function in $|z|<1$. Therefore

$$
\int_{0}^{2 \pi} f_{\alpha}\left(z, e^{i s}\right) d s \leq \max _{t} \int_{0}^{2 \pi} f_{\alpha}\left(e^{i t}, e^{i s}\right) d s
$$

Since

$$
\begin{aligned}
f_{\alpha}\left(e^{i t}, e^{i s}\right) & =\left|\Re\left[e^{i(\alpha+s)}\left(e^{i t}-e^{i s}\right)^{n-1}\right]\right|^{q}\left|e^{i t}-e^{i s}\right|^{2 q-2} \\
& =\left|\Re\left[e^{i(\beta+u)}\left(1-e^{i u}\right)^{n-1}\right]\right|^{q}\left|1-e^{i u}\right|^{2 q-2},
\end{aligned}
$$

for $u=s-t$ and $\beta=\alpha+n t$ we obtain the second statement of the lemma.

Proof of Theorem 1.2. As above, we have

$$
\begin{aligned}
& \left|\Re\left[e^{i(\alpha+s)}\left(e^{i t}-e^{i s}\right)^{n-1}\right]\right|^{q} \\
& \quad=2^{(n-1) q / 2}\left|\cos \left[\beta-\frac{\pi}{2}(n-1)+u \frac{n+1}{2}\right]\right|^{q}(1-\cos u)^{(n-1) q / 2}
\end{aligned}
$$

and

$$
\left|1-e^{i u}\right|^{2 q-2}=2^{q-1}(1-\cos u)^{q-1} .
$$

In view of Lemma 2.2 we have

$$
I_{\alpha}(r)=2^{(n+1) q / 2-1}\left(1-r^{2}\right)^{1-(1+n) q} F_{q}(\beta),
$$

where

$$
F_{q}(\beta)=\int_{0}^{2 \pi}(1-\cos u)^{\frac{(n+1) q-2}{2}}\left|\cos \left[u \frac{n+1}{2}+\beta-\frac{\pi}{2}(n-1)\right]\right|^{q} d u .
$$

Moreover

$$
F_{q}(\beta)=2^{(n+1) q / 2} \int_{0}^{\pi}\left|\phi_{\beta}(v)\right|^{q} d v
$$

where

$$
\phi_{\beta}(v)=\sin ^{(n+1)-2 / q} v \cos \left[v(n+1)+\beta-\frac{\pi}{2}(n-1)\right] .
$$

It can be proved easily that $F_{q}(\beta)=F_{q}(\pi-\beta)$. The last fact implies that it is enough to find the maximum in $[0, \pi / 2]$.

3. The CASE $q=1$ AND the PRoof of Theorem 1.4

We divide the proof into two cases and use the notation $F=F_{q}$. 
3.1. The odd $n$. For $n=2 m-1$ and $q=1$ we have

$$
F(\beta)=2^{m} \int_{0}^{\pi} \sin ^{n-1} v|\cos [(n+1) v+\beta]| d v .
$$

Then

$$
\frac{d}{d x} \frac{\cos (\beta+n x) \sin ^{n} x}{n}=(-1)^{m-1} \phi_{\beta}(x)=\sin ^{n-1} x \cos [\beta+(n+1) x] .
$$

Since $F$ is $\pi$-periodic we can assume that $-\pi / 2 \leq \beta \leq \pi / 2$. Assume that $0 \leq$ $\beta<\pi / 2$ (the second case can be treated similarly). Then $\cos [\beta+2 m x] \geq 0$ and $0 \leq x \leq \pi$ if and only if one of the following relations hold

- $\beta \leq \beta+2 m x<\frac{\pi}{2}$

- $-\frac{\pi}{2}+2 k \pi<\beta+2 m x<\frac{\pi}{2}+2 k \pi$, for $1 \leq k \leq m-1$ or

- $-\frac{\pi}{2}+2 m \pi<\beta+2 m x<\beta+2 m \pi$

or, what is the same, if:

- $a_{0}=0<x<b_{0}=\frac{\pi-2 \beta}{4 m}$

- $a_{k}:=\frac{-\pi+4 k \pi-2 \beta}{4 m}<x<b_{k}:=\frac{\pi+4 k \pi-2 \beta}{4 m}$, for $1 \leq k \leq m-1$ or

- $a_{m}:=\frac{-\pi+4 m \pi-2 \beta}{4 m}<x<b_{m}:=\pi$.

From 3.1 for

$$
g_{n}(x)=\frac{\cos (\beta+n x) \sin ^{n} x}{n},
$$

because $g_{n}(\pi)-g_{n}(0)=0$, we have

$$
\begin{aligned}
F(\beta) & =2^{m} \int_{0}^{\pi}\left|\phi_{\beta}(v)\right| d v \\
& =2 \cdot 2^{m} \int_{0 \leq v \leq \pi: \phi_{\beta}(v) \geq 0} \phi_{\beta}(v) d v-2^{m} \int_{0}^{\pi} \phi_{\beta}(v) d v \\
& =2^{m+1} \int_{0 \leq v \leq \pi: \phi_{\beta}(v) \geq 0} \phi_{\beta}(v) d v .
\end{aligned}
$$

Therefore

$$
\frac{F(\beta)}{2^{m+1}}=\sum_{k=0}^{m}\left[g_{n}\left(b_{k}\right)-g_{n}\left(a_{k}\right)\right]=g_{n}\left(b_{0}\right)-g_{n}\left(a_{m}\right)+\sum_{k=1}^{m-1}\left[g_{n}\left(b_{k}\right)-g_{n}\left(a_{k}\right)\right] .
$$

But for $1 \leq k \leq m$

$$
g_{n}\left(b_{k}\right)=\frac{\sin ^{2 m} b_{k}}{n} \text { and } g_{n}\left(a_{k}\right)=-\frac{\sin ^{2 m} a_{k}}{n} .
$$

Therefore

$$
F(\beta)=\frac{2^{m+1}}{n} \sum_{k=1}^{2 m} \sin ^{2 m}\left[\frac{-2 \beta+(2 k-1) \pi}{4 m}\right]=\frac{2^{m+1}}{n} \sum_{k=1}^{2 m} \sin ^{2 m}\left[\frac{\gamma+k \pi}{2 m}\right],
$$

where $\gamma=-\pi / 2-\beta$. Now by invoking [2, Lemma 3.5], we have

$$
f(\beta):=\sum_{k=1}^{2 m} \sin ^{2 m}\left[\frac{\gamma+k \pi}{2 m}\right]=\frac{2}{B\left(\frac{1}{2}, m\right)},
$$

and therefore

$$
F(\beta)=\frac{4 m}{n 2^{m}}\left(\begin{array}{c}
2 m \\
m
\end{array}\right)
$$


3.2. The even $n$. For $n=2 m$ and $q=1$

$$
F(\beta)=2^{(2 m+1) / 2} \int_{0}^{\pi} \sin ^{n-1} v|\sin [v(n+1)+\beta]| d v .
$$

Let

Since

$$
g(x)=\frac{\sin (\beta+n x) \sin ^{n} x}{n} .
$$

from (3.3) and

$$
\frac{d}{d x} g(x)=(-1)^{m-1} \phi_{\beta}(x)=\sin ^{n-1} x \sin [\beta+(n+1) x],
$$

$$
\begin{aligned}
F(\beta) & =2^{(n+1) / 2} \int_{0}^{\pi}\left|\phi_{\beta}(v)\right| d v \\
& =2 \cdot 2^{(n+1) / 2} \int_{0 \leq v \leq \pi: \phi_{\beta}(v) \geq 0} \phi_{\beta}(v) d v-2^{(n+1) / 2} \int_{0}^{\pi} \phi_{\beta}(v) d v \\
& =2^{(n+1) / 2+1} \int_{0 \leq v \leq \pi: \phi_{\beta}(v) \geq 0} \phi_{\beta}(v) d v,
\end{aligned}
$$

we obtain

$$
\frac{F(\beta)}{2^{(n+1) / 2+1}}=g\left(\frac{-\beta+\pi}{1+n}\right)+\sum_{k=1}^{m}\left(g\left(\frac{-\beta+(1+2 k) \pi}{1+n}\right)-g\left(\frac{-\beta+2 k \pi}{1+n}\right)\right) .
$$

After some elementary transformations we obtain

$$
2^{-(n+1) / 2} m F(\beta)=\sum_{k=1}^{n+1} \sin ^{1+n}\left[\frac{-\beta+k \pi}{1+n}\right] .
$$

This finishes the proof of Theorem 1.4 .

\section{Appendix}

In this section we include a possible strategy how to determine the maximum of the function $F$ in $[0, \pi / 2]$ provided that $n=2 m$ is an even integer. First of all

$$
2^{-(2 m+1) / 2} m F^{\prime}(\beta)=-\sum_{k=1}^{1+2 m} \cos \left[\frac{-\beta+k \pi}{1+2 m}\right] \sin ^{2 m}\left[\frac{-\beta+k \pi}{1+2 m}\right] .
$$

Let

$$
h_{k}(\beta)=\cos \left[\frac{-\beta+k \pi}{1+2 m}\right] \sin ^{2 m}\left[\frac{-\beta+k \pi}{1+2 m}\right] .
$$

Then for $1 \leq k \leq 2 m, h_{k}(0)+h_{2 m+1-k}(0)=0, h_{2 m+1}(0)=0$ and $h_{k}(\pi / 2)+$ $h_{2 m+2-k}(\pi / 2)=0$ and $h_{m+1}(\pi / 2)=0$. It follows that

$$
F^{\prime}(0)=F^{\prime}\left(\frac{\pi}{2}\right)=0 .
$$

Thus 0 and $\pi / 2$ are stationary points of $F$.

It can be shown that for $\gamma=\beta+\pi / 2$ and for $\pi / 2 \leq \gamma \leq \pi$

$$
\frac{m F(\beta)}{2^{(n+1) / 2}}=\sum_{j=0}^{m} \frac{(-1)^{j}}{2^{n}}\left(\begin{array}{c}
1+n \\
m-j
\end{array}\right) \frac{\cos \left[\frac{(1+2 j) \gamma}{1+n}\right]}{\sin \left[\frac{(1+2 j) \pi}{2(1+n)}\right]}+2 \sin ^{1+n}\left[\frac{\gamma-\pi / 2}{1+n}\right] .
$$


We expect that the formula (4.1) can be more useful than (3.4) in finding the maximum of the function $F(\beta)$, however it seems that the corresponding problem is hard. By using the software "Mathematica 8" we can see that $F(0)<F(\beta)<$ $F(\pi / 2)$ provided that $n=4 k$ and $0<\beta<\pi / 2$ and $F(\pi / 2)<F(\beta)<F(0)$ provided that $n=4 k+2$ and $0<\beta<\pi / 2$ (cf. Conjecture 4.1). We do not have a proof of the previous fact but we include in this paper the following special cases.

4.1. The case $m=1(n=2)$ and $q=1$. We have

$$
F(\beta)=\frac{\sqrt{2}}{2}\left(3 \sqrt{3} \cos \frac{\beta}{3}+4 \sin ^{3} \frac{\beta}{3}\right)
$$

and

$$
F^{\prime}(\beta)=-\frac{\sin \frac{\beta}{2}\left(\sqrt{3}-2 \sin \frac{2 \beta}{3}\right)}{4} .
$$

Thus $F^{\prime}(\beta)=0$ if and only if $\beta=0$ or $\beta=\frac{\pi}{2}$. The minimum of $F(\beta)$ is $F\left(\frac{\pi}{2}\right)=\frac{5 \sqrt{2}}{2}$ and the maximum is $F(0)=\frac{3 \sqrt{6}}{2}$.

4.2. The case $m=2(n=4)$ and $q=1$. In this case

$$
F(\beta)=\frac{\sqrt{2}}{8}\left(10 \sqrt{5+2 \sqrt{5}} \cos [\beta / 5]-5 \sqrt{5-2 \sqrt{5}} \cos [3 \beta / 5]+16 \sin [\beta / 5]^{5}\right) .
$$

Then it can be proved that $F$ is increasing in $[0, \pi / 2]$ and

$$
F(0)=5 / 4 \sqrt{12.5+\sqrt{5}} \approx 4.79845<F(\pi / 2)=\sqrt{381 / 32+5 \sqrt{5}} \approx 4.80485 .
$$

By differentiating the subintegral expression (1.7) w.r.t $\beta$ we can easily conclude that $\beta=0$ and $\beta=\pi / 2$ are stationary points of $F$ provided that $q \geq 1$ and $n \in \mathbf{N}$. This and some experiments with the software "Mathematica 8" leads to the following conjecture

Conjecture 4.1. Denote by $[a]$ the integer part of $a$. We conjecture that:

- $F_{q}$ is decreasing on $\left[0, \frac{\pi}{2}\right]$ for $q>2$

- $F_{q}$ is nondecreasing (nonincreasing) on $\left[0, \frac{\pi}{2}\right]$ for $q \leq 2$ and $\left[\frac{(n+1) q}{2}\right]$ is an even (odd) integer.

\section{Appendix B}

In this appendix we offer some numerical estimation that confirm tha our conjecture is true, at least for $q=1$. Whereas as is observed in (3.2) for $0 \leq \beta \leq \pi$

$$
f(\beta)=f_{n}(\beta)=\sum_{k=1}^{s} \sin ^{s} \frac{-\beta+k \pi}{s}
$$

is the constant $2 / B\left(\frac{1}{2}, \frac{s}{2}\right)$ for even $s$, if $s=n+1$ is odd the maximum exceeds $2 / B\left(\frac{1}{2}, \frac{s}{2}\right)$ by a tiny amount that is very nearly

$$
\frac{4}{\pi} \frac{1}{s+2} \frac{2}{s+4} \frac{3}{s+6} \cdots \frac{s}{3 s}=\frac{4}{\pi} s ! \frac{s ! !}{(3 s) ! !}=(27+o(1))^{-s / 2}
$$

for large $s$. Here and later we use " $u$ !!" only for positive odd $u$ to mean the product of all odd integers in $[1, u]$; that is, $u ! !:=u ! /\left(2^{v} v !\right)$ where $u=2 v+1$. In order to outline the proof of the last statement we do as follows. 
For $s=2 m+1$ we define the function $g$ as follows

$$
g(x):=f\left(x+\frac{\pi}{2}\right)=g(-x)=-g(x+s \pi)
$$

which has a finite Fourier expansion in cosines of odd multiples of $X:=x / s$, namely

$$
f(x)=(-1)^{m} 2^{-s} \sum_{j=0}^{s}(-1)^{j}\left(\begin{array}{l}
s \\
j
\end{array}\right) \frac{\cos t X}{\sin \frac{\pi t}{2 s}}
$$

where $t=s-2 j$. We deduce from (5.1) that

$$
f(\beta)-f(\beta+\pi)=2 \sin ^{s}(\beta / s),
$$

from which it follows that $g(x)$ is maximized somewhere in $|x| \leq \pi / 2$, but that changing the optimal $x$ by a small integral multiple of $\pi$ reduces $g$ by a tiny amount; this explains the near-maxima we observed at $x= \pm \pi$ for $2 \mid m$, and indeed the further oscillations for both odd and even $m$ that we later noticed as $s$ grows further.

This also suggests that in and near the interval $|x| \leq \pi / 2$ our function $g$ should be very nearly approximated for large s by an even periodic function $\tilde{g}(x)$ of period $\pi$. We next outline the derivation of such an approximation, with $\tilde{g}$ having an explicit cosine-Fourier expansion

$$
\tilde{g}(x)=g_{0}+g_{1} \cos 2 x+g_{2} \cos 4 x+g_{3} \cos 6 x+\cdots
$$

where $g_{0}=2 / B\left(\frac{1}{2}, \frac{s}{2}\right)$ and, for $l>0$,

$$
g_{l}=(-1)^{m+l-1} \frac{4}{\pi} \frac{s !}{2 l+1} \frac{((2 l-1) s) ! !}{((2 l+1) s) ! !}
$$

with the double-factorial notation defined as above. Thus

$$
\tilde{g}(x)=g_{0}+(-1)^{m} \frac{4 s !}{\pi}\left(\frac{s ! !}{(3 s) ! !} \cos 2 x-\frac{1}{3} \frac{(3 s) ! !}{(5 s) ! !} \cos 4 x+\frac{1}{5} \frac{(5 s) ! !}{(7 s) ! !} \cos 6 x \mp \cdots\right) .
$$

For large $s$, this is maximized at $x=0$ or $x= \pm \pi / 2$ according as $m$ is even or odd. Since we already know by symmetry arguments that $g^{\prime}(0)=g^{\prime}( \pm \pi / 2)=0$, this point or points will also be where $g$ is maximized, once it is checked that $g-\tilde{g}$ and its first two derivatives are even tinier there.

The key to all this is the partial-fraction expansion of the factor $1 / \sin (\pi t / 2 s)$ in the Fourier series of $g$, obtained by substituting $\theta=\pi t / 2 s$ into

$$
\frac{1}{\sin \pi \theta}=\frac{1}{\pi} \sum_{l=-\infty}^{\infty} \frac{(-1)^{l}}{\theta-l}
$$

with the conditionally convergent sum interpreted as a principal value or Cesáro limit etc. On the other hand the main term, for $l=0$, yields the convolution of $\cos ^{s}(x / s)$ with a symmetrical square wave, which is thus maximized at $x=0$ and almost constant near $x=0$; we identify the constant with $2 / B\left(\frac{1}{2}, \frac{s}{2}\right)$ using the known product formula for

$$
\int_{-\pi / 2}^{\pi / 2} \cos ^{s} X d X
$$

The new observation is that each of the error terms $(-1)^{l} /(\theta-l)$ likewise yields the convolution with a square wave of

$$
(-1)^{l} \cos (2 l x) \cos ^{s}(x / s) \text {. }
$$


If we approximate this square wave with a constant, we get the formula for $g_{l}$ displayed above, via the formula for the $s$-th finite difference of a function $1 /\left(j_{0}-j\right)$. The error in this approximation is still tiny (albeit not necessarily negative) because $\cos ^{s}(x / s)$ is minuscule when $x$ is within $\pi / 2$ of the square wave's jump at $\pm \pi s / 2$.

We've checked these approximations numerically to high precision (modern computers and gp make this easy) for $s$ as large as 100 or so, in both of the odd congruence classes mod 4 , and it all works as expected; for example, when $s=99$ we have $f(0)-g_{0}=2.57990478176660 \ldots \cdot 10^{-70}$, which almost exactly matches the main term $g_{1}=(4 / \pi) 99$ ! $99 ! ! / 297 !$ ! but exceeds it by $5.9110495 \ldots \cdot 10^{-102}$, which is almost exactly $g_{2}=(4 / \pi) 99 ! 297 ! ! /(3 \cdot 495 ! !)$ but too large by $7.92129 \ldots \cdot 10^{-120}$, which is almost exactly $g_{3}=(4 / \pi) 99$ ! $495 ! ! /(5 \cdot 693 ! !)$, etc.; and likewise for $s=101$ except that the maximum occurs at $\beta=\pi / 2$ and is approximated by an alternating sum $g_{1}-g_{2}+g_{3} \ldots$ (actually here this approximation is exact because $x=0$ ).

Acknowledgement. We are thankful to the referee for some corrections and comments, that have improved this paper.

\section{REFERENCES}

[1] S. Axler, P. Bourdon And W. Ramey: Harmonic function theory. Springer Verlag New York 1992.

[2] M. Beck, M. Halloran: Finite Trigonometric Character Sums Via Discrete Fourier Analysis. International Journal of Number Theory 6, no. 1 (2010), 51-67

[3] F. Colonna: The Bloch constant of bounded harmonic mappings. Indiana Univ. Math. J. 38 (1989), no. 4, 829-840.

[4] P. Duren, Theory of $H^{p}$ spaces. Pure and Applied Mathematics, Vol. 38 Academic Press, New York-London 1970 xii+258 pp.

[5] J. Garnett: Bounded analytic functions. Pure and Applied Mathematics, 96. Academic Press, Inc., New York-London, 1981. xvi+467 pp.

[6] D. Khavinson: An extremal problem for harmonic functions in the ball. Canad. Math. Bull., 35 (1992), 218-220.

[7] D. Kalaj and M. Vuorinen: On harmonic functions and the Schwarz lemma, Proc. Amer. Math. Soc. 140 (2012) 161-165.

[8] D. Kalaj, M. Marković: Optimal estimates for the gradient of harmonic functions in the unit disk, Complex analysis and operator theory: DOI: 10.1007/s11785-011-0187, arXiv:1012.3153

[9] G. KResin and V. MaZ'ya: Sharp real part theorems for higher order derivatives, J. of Math. Sciences, 181 (2012), no. 2, pp 107-125.

[10] G. Kresin and V. Maz'ya: Sharp Real-Part Theorems A Unified Approach, Lecture Notes in Mathematics, Springer, Volume 1903, 2007.

[11] A. J. Macintyre And W. W. Rogosinski: Extremum problems in the theory of analytic functions. Acta Math. 82, 1950, 275 - 325.

[12] M. PAVlović: Introduction to function spaces on the disk. 20. Matematički Institut SANU, Belgrade, 2004. vi+184 pp.

[13] S. Ruscheweyh: Two remarks on bounded analytic functions, Serdica, 11, s 2, 200-202, (1985).

University of Montenegro, Faculty of Natural Sciences and Mathematics, Cetinjski PUt B.B. 81000 Podgorica, Montenegro

E-mail address: davidk@ac.me

Department of Mathematics, Harvard University, Cambridge, MA 02138

E-mail address: elkies@math.harvard.edu 